\title{
SYMBIOTIC SIMULATION FOR BUSINESS PROCESS RE-ENGINEERING IN HIGH-TECH MANUFACTURING AND SERVICE NETWORKS
}

\author{
Malcolm Y. H. Low \\ Stephen J. Turner \\ Ding Ling \\ Hai L. Peng \\ Nanyang Technological University \\ Nanyang Avenue \\ Singapore 639798, SINGAPORE \\ Peter Lendermann \\ D-SIMLAB Technologies Pte Ltd \\ 30 Biopolis Street, Matrix \\ Singapore 138671, SINGAPORE
}

\author{
Lai P. Chan \\ Singapore Institute of Manufacturing Technology \\ 71 Nanyang Drive \\ Singapore 638075, SINGAPORE
}

\begin{abstract}
In today's highly competitive business environment, the speed of a company's response to changes by adapting its own business processes is vital to its survival. In this paper, we propose a symbiotic simulation system that can monitor the real-world operations of high-tech manufacturing and service networks, carry out what-if analysis and optimization on service-oriented based business workflow, and dynamically deploy the optimized business workflow onto the real-world operations. A case study of an aerospace spare parts logistics system was carried out to investigate the viability of the system.
\end{abstract}

\section{INTRODUCTION}

In today's highly competitive business environment, the speed with which a company responds to changes in its business environment by adapting and enhancing its own business processes is vital to its survival. However, experimentation with the real system is too disruptive and costly and often is not possible at all. One widely used technique in industries is to carry out virtual experimentation using simulation to aid in strategic decision making on their operational processes. However, in high-tech manufacturing and service networks, the nature of the fast changing business environments makes it difficult to develop, validate and maintain the various models representing the manufacturing and logistics systems (Lendermann et al. 2007).
Symbiotic simulation is an emergent technology proposed by the Parallel and Distributed Simulation working group at the 2002 Dagstuhl seminar on Grand Challenges for Modelling and Simulation (Fujimoto et al. 2002). Essentially, a symbiotic simulation system consists of a simulation model interacting with the physical system in a mutually beneficial way. The simulation system benefits from the continuous supply of the latest data and the automatic validation of its simulation outputs, whereas the physical system benefits from optimized performance obtained from the analysis of simulation experiments. A symbiotic integration of the real-time physical system and the corresponding decision support module will enable prompt response to be carried out to handle abrupt changes in the physical system.

Increasingly, businesses are leveraging on the Service Oriented Architecture (SOA) approach by providing individual business processes as stand-alone web services and business workflow as a collection of these web services. In this paper, we describe our work in the development of a prototype symbiotic simulation system using the IBM WebSphere Business Integration (WBI) Suite to carry out dynamic business process re-engineering using a case study for an aerospace spare parts logistics system.

The rest of the paper is organized as follows. Section 2 describes some related work in the area of symbiotic simulation systems. Section 3 describes the aerospace spare parts logistics case study used in this paper. A brief introduction to the IBM WBI Suite used in our work is given in Section 4. The architecture of our proposed symbiotic 
simulation system is described in Section 5. Section 6 presents a set of experimental results on the aerospace spare parts logistics case study. Section 7 concludes the paper and outlines some future work.

\section{RELATED WORK}

In our previous work, we demonstrated the use of a symbiotic simulation system in a case study where software agents are used in the monitoring, optimization and control of the manufacturing operations of a semiconductor assembly and test factory (Low et al. 2005). One drawback in this previous work carried out is that the business process logic is embedded in the simulation model and it is difficult to implement changes to the real world business process. In this paper, we describe an approach to separate the business process logic from an aerospace spare components logistics simulation. Symbiotic simulation can then be used to analyze and carry out re-engineering of the business processes associated with high-tech manufacturing and service networks.

One of the key components of a symbiotic simulation system is the adaptation of the simulation model based on the optimization requirements as well as feedback from real-world data. Reynolds and his team at the University of Virginia have developed the technique COERCE for simulation reuse as part of the Dynamic Data-Driven Application System (DDDAS) project funded by the National Science Foundation. In Carnahan, Reynolds, and Brogan (2004) and Loitiere, Brogan, and Reynolds (2004), the authors describe the input and runtime parameters of an existing simulation model that can be coerced to facilitate experimentation and composibility of simulation components comprising the DDDAS. In Liu, Reynolds, and Brogan (2006), the authors describe the use of abstraction in the optimization and verification of simulation coercion.

In this paper, an aerospace spare parts logistics case study is used to demonstrate how symbiotic simulation can be applied to business process re-engineering. The existing business processes of the aerospace spare parts logistics operation are first exposed as Web services. These Web services (and any new services developed later) can then be dynamically assembled to form new and improved business workflow using the Business Process Execution Language (BPEL) for Web services. BPEL is an XMLbased language that allows the specification of business processes and how they relate to Web services (Leymann and Roller 2002). BPEL specifies how a business process makes use of Web services to achieve its goal, as well as the Web services that are provided by a business process. A BPEL business process orchestrates the Web services of its partners and supports the specification of business protocols between partners. In our previous work, we have demonstrated how BPEL can be used to support the configuration, deployment and execution of a large-scale distributed simulation system (Low and Turner 2006).

\section{AEROSPACE SPARE COMPONENTS LOGISTICS CASE STUDY}

The aerospace spare components logistics case study used in our work is designed to explore the possibility of carrying out business process re-engineering using a symbiotic simulation system. In the aerospace industry, the availability of spare components is a critical issue for ensuring safety and punctuality of flights. Due to the high cost of aerospace spare components, airlines prefer to pool resources and maintain minimum inventory level instead of stocking an excessive number of spare components in each airport. Thus, a resource management business application is developed to manage the inventory level of all the airports.

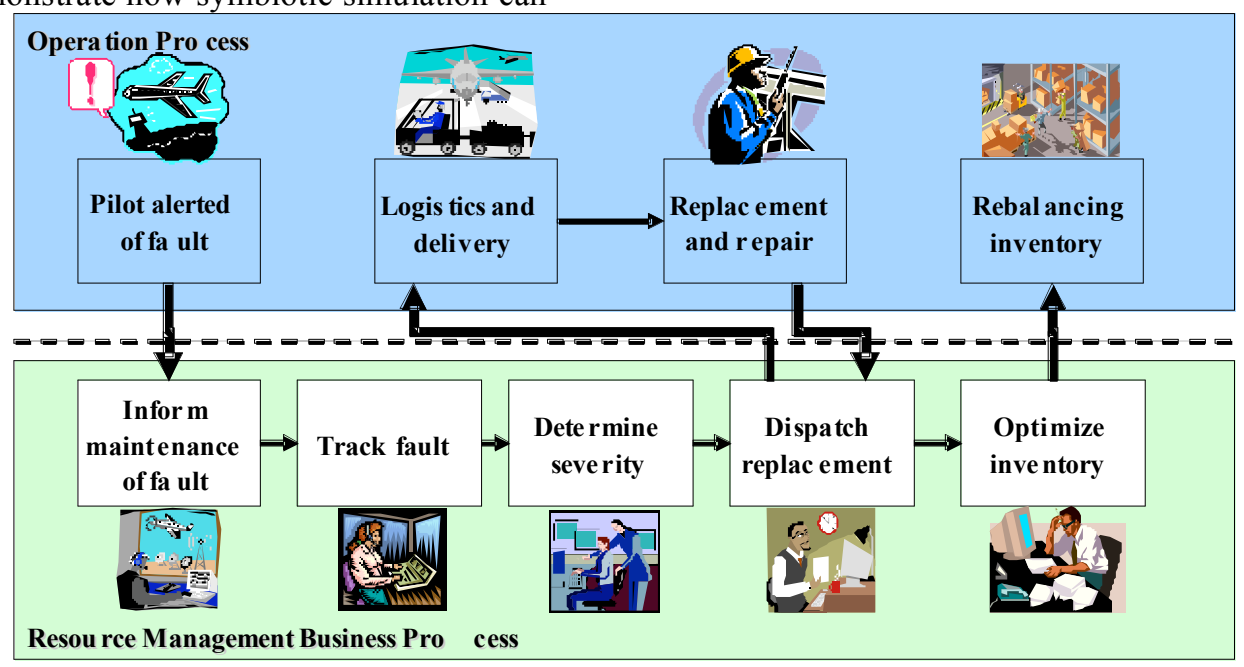

Figure 1: Business and operational process for aerospace spare component logistics. 
Aircraft component faults may be detected at different phases of the flight. When a faulty component is reported, the severity of the fault is analyzed and determined to decide where to search for the spare component for replacement in the inventory. The inventory of each airport can be either located at the line station (close to the airstrip) or the warehouse (near the airport). The local line station and warehouse of the destination airport will first be searched. If the component is not available in the local inventory, inventories of other airports in the same region or worldwide may be searched. This will also require looking for an appropriate service flight to carry the spare component from the available inventory to the destination airport. Once the replacement is found, it will be dispatched for replacement. The faulty component will be sent for repair, after which it can be returned to the inventory from which the replacement is withdrawn. The number of components in each inventory may need to be re-balanced accordingly to obtain an optimal inventory level.

\subsection{Resource Management Business Process Work Flow}

Figure 1 shows the operational and business processes of the aerospace spare components logistics operation. The resource management business application is a workflow that consists of the following five processes:

i) Inform the Maintenance Control Center (MCC) of flight fault;

ii) track all fault detection requests;

iii) determine the impact of the fault;

iv) arrange for spare component to replace faulty component;

v) re-balance the inventory across all airports.

To ensure adequate service level, the number of spare components across all airports has to be continuously optimized and re-balanced during actual operation. Simulation can be used to determine the optimal number of spare components under various scenarios and resource management algorithms. Traditionally, using this approach, the business process is typically built into the simulation model. To evaluate the impact of any modification to the business process, the simulation model has to be reconstructed, which is often a lengthy process. Also, changes to the "live" business process cannot be effected on-thefly. In this paper, we propose an approach whereby the business process is separate from the simulation model, and modifications can be made to the business process directly without changing the simulation model.

\subsection{Resource Management Application as a BPEL Web Service}

Web services allow easy configuration of components to create dynamic applications with flexible data models that allow gradual implementation. BPEL provides a flexible and transparent mechanism to orchestrate Web service components to form a business process. Using BPEL, the overall time and effort will be reduced significantly for reconfiguration and enhancement of the application when changes need to be made.

To achieve the required flexibility for business process re-engineering, we first separated the resource management business logic from the simulation model. Webservice based business process application components are used to develop the business application. The entire resource management business process flow is decomposed into the five atomic and decoupled tasks described above. Each of these tasks is implemented and deployed as a Web service.

BPEL is then used to orchestrate and link together these five Web services to form a complete workflow from notification of flight fault to the re-balancing of spare components. The resource management business application is then integrated with a simulation model representing a flight operation process as in a distributed simulation environment. To study the business re-engineering process and analyze how the business process performs under different conditions, the business process flow can be adjusted and changed by reordering the BPEL flow. Different BPEL templates will be created representing variations of the business process. Subsequent analysis to determine the optimal inventory and to find inventory policies for differentiated service levels can be conducted.

\section{IBM WEBSPHERE BUSINESS INTEGRATION (WBI) SUITE}

In our system, the IBM WBI Suite (IBM 2007) is used to realize the resource management business workflow and the individual business Web services. The IBM WBI suite provides an integrated environment to model, orchestrate, deploy and monitor business processes using Web services and standards such as BPEL. Its business process management (BPM) capabilities allow an enterprise to respond adeptly to changes in operational conditions. The modelling and monitoring abilities also enable continuous business process improvement. The different components of the WBI Suite are as follows:

- WebSphere Business Modeler: targeted at business analysts to help capture business design. The Modeler can be used for documentation and compliance purposes, providing a visual and textual representation of processes, information, organization, resources, classifiers, and business meas- 
urements that can be shared across an organization. In this study, the WBI Modeler is used to create the template for the three BPEL process flows described in section 6.1.

- WebSphere Integration Developer (WID): an Eclipse-based tool designed to help create business process flows, state machines, and business rules. WID simplifies integration with its Service Component Architecture (SCA). SCA uses BPEL for assembling business process tasks into workflows, which can then be deployed to the WebSphere Process Server. WID can also directly import business models from the IBM Business Modeler.

- WebSphere Process Server (WPS): the primary hosting environment for business processing. Built on the WebSphere Enterprise Service Bus, WPS includes support for both BPEL based process flows and business state machines. WPS also supports the integration of business rules in process and service selection.

- WebSphere MQ: enables programs to communicate with one another across a network of heterogeneous components (processors, operating systems, subsystems, and communication protocols) using a consistent Application Programming Interface (API). Applications designed and written using this interface are known as message queuing applications, because they use the messaging and queuing style.

- WebSphere Message Broker: an information broker that allows business information, in the form of messages, to flow between disparate applications and across multiple hardware and software platforms. Business rules can be applied to the data that is flowing through the Message Broker in order to route, store, retrieve, and transform the information through the definition of message flows.

- WebSphere Business Monitor: monitors business processes in real time, providing a live visual display of business process status. The Monitor complements the WebSphere Business Modeler. It helps in creating dashboards for visualizing the performance of a business, based on the key performance indicators that are identified in a business design. This can be used to track time, cost, and resources used in processes. The Business Monitor provides tools that enable users to set situational triggers and notifications of potential bottlenecks or workload imbalances.

\section{SYMBIOTIC SIMULATION SYSTEM}

Figure 2 shows the architecture of a generic symbiotic simulation system for business process re-engineering. It comprises of a discrete-event simulator and a middleware to bridge the business/operation logic to the simulation. The framework serves as an integrated decision support tool to continuously test, verify and optimize changes and improvement to the business process. A monitoring tool (e.g. WBI Business Monitor) allows the dynamic visualization of the entire business and provides real-time data that enables more accurate simulation analysis to be carried out to improve business processes. The improvement can be viewed in the simulation world using the monitoring tool and a comparison with the real system can be drawn. Furthermore, it allows tracking of the success of changes made in the business based on identified business metrics. In this way, the business application becomes a "live" system emulating the real world scenario and will evolve and grow with business needs. The existing business process can be reused for simulation and new processes explored through simulation can be deployed directly back to the "live" system.

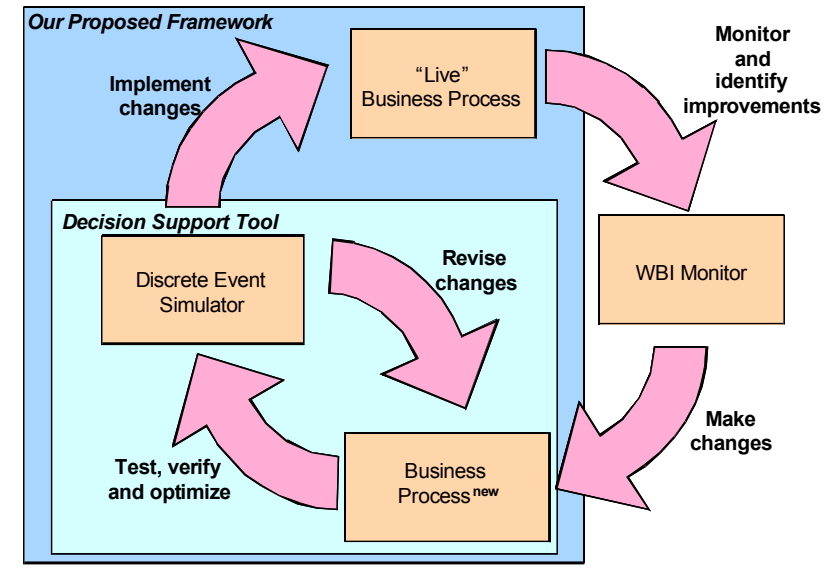

Figure 2: Generic symbiotic simulation system.

Figure 3 shows the overall architecture of our proposed symbiotic simulation system. It provides an overview of how the individual software components, both commercially and in-house developed, work together in order to create a simulation system with the BPEL resource management business application.

\subsection{CSPE Simulator}

In our previous work in investigating interoperability issues between commercial simulators, we developed a simulation of flight cycles and generation of aircraft component faults using the COTS Simulation Package (CSP) Emulator (CSPE). CSPE is a simple pipeline simulation engine that is intended to emulate the functionality and in- 


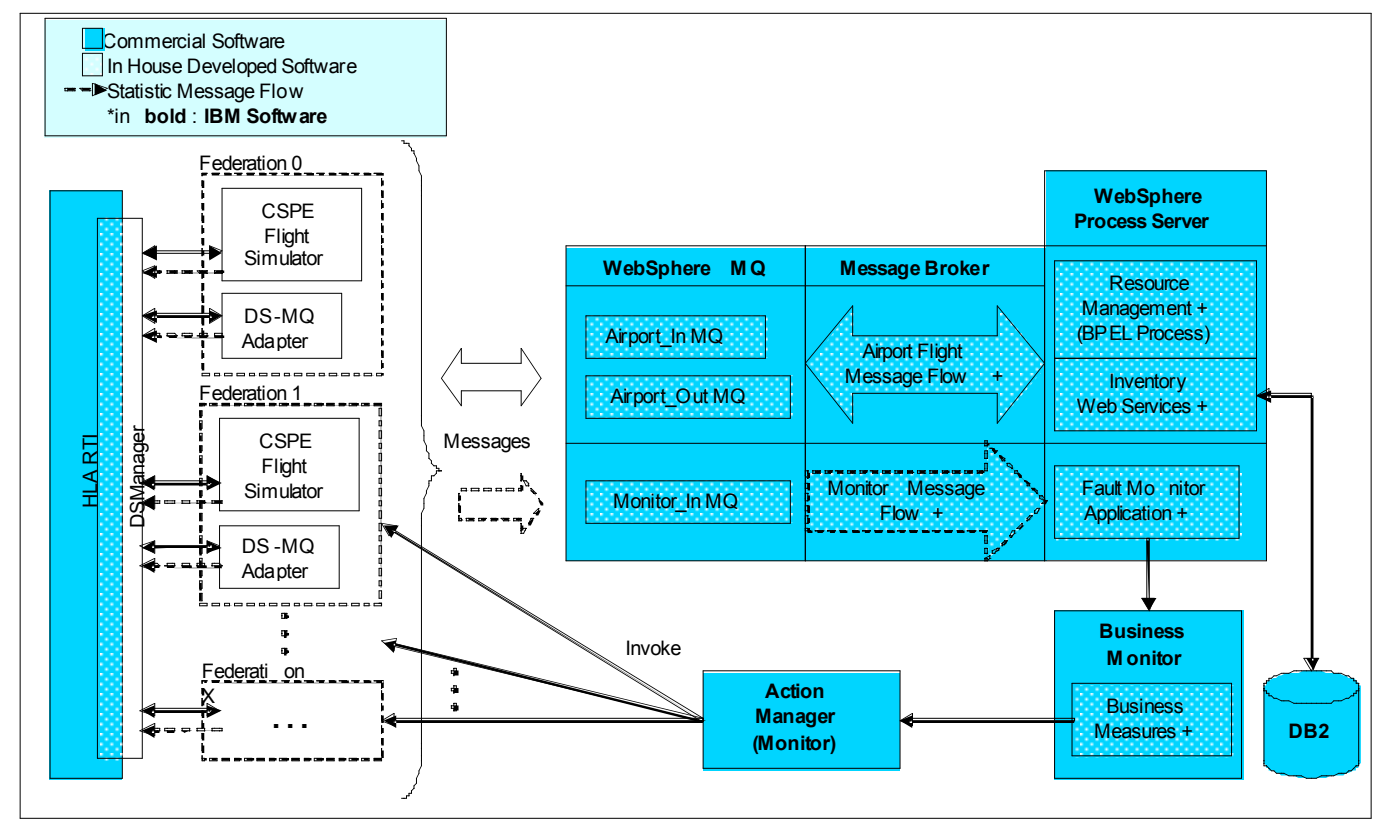

Figure 3: Architecture of symbiotic simulation system.

terface of a CSP (Wang et al. 2005). As our system is a prototype, the real world operational process of the aerospace spare component logistics described in Figure 1 is emulated using a CSPE flight simulator. During simulation optimization, CSPE flight simulators are also use to simulate operational processes in what-if scenarios.

\subsection{DSManager and HLA}

To allow interaction between the CSPE flight simulator and the resource management BPEL application, the High Level Architecture (HLA) distributed simulation standard is used. HLA is an IEEE standard (IEEE 1516 2000) for the infrastructure needed for large-scale distributed simulation. The HLA defines the rules and specifications to support reusability and interoperability of different simulators (Kuhl, Weatherly, and Dahmann 1999). In HLA terminology, a simulation component is referred to as a federate. A federation is then a set of federates working together to achieve a given goal. Each federate interacts with one another over the Runtime Infrastructure (RTI) (DMSO 2002). A set of simulation models developed independently can be put together to form a larger simulation (or federation). Using the HLA, each participating federate in the federation can define the objects and interactions that are shared with others in its simulation object model (SOM), but its internal behavior (and data) is completely invisible to the outside world.

The DSManager (Distributed Simulation Manager) is a software library developed by Wang et al. (2005) at Nanyang Technological University to study synchronization and interoperability issues in distributed simulations. It provides a generic interface for a set of federate-related functions to be invoked by CSPs. The DSManager hides the complexity of the time management issues in the HLA from CSP users. In our system, the CSPE flight simulator forms a federate and the resource management BPEL, through the DS-MQ Adaptor (see section 5.3) forms another federate. The two federates communicate with each other through the DSManager/HLA and form a federation.

\subsection{DS-MQ Adaptor}

The DS-MQ Adapter was developed in our work to serve as an interfacing federate representing the resource management business module in the distributed simulation. It receives component fault entities generated by the flight simulator through the DSManager/HLA interface and places them on a predefined WebSphere Message Queue (MQ). The fault entities in MQ will be sent using the WebSphere Message Broker to the resource management application, which is deployed as a BPEL business process and exposed as a Web service on the WebSphere Process Server. The WebSphere Message Broker is used in our work to provide a message routing service which routes messages in MQ based on predefined message flows. In a real world application, messages from the MQ may be routed to several other business applications within an enterprise.

The business application finds a replacement component from the inventory (DB2 Database) for the faulty one, and returns the replacement component details to a Message Queue through the Message Broker. The DSMQ Adapter then reads the replacement component from the return Message Queue, and delivers it back to the 
CSPE simulator through the DSManager/HLA. This cycle of fault detection and fault servicing repeats until the end of the simulation.

\subsection{Business Monitor}

The success or failure of each fault servicing cycle is determined by the time taken for the replacement component to be made available. If this exceeds the departure time of the flight that has the faulty component, the servicing has failed. Thus, a performance indicator "FillRate Percentage" is defined to measure the percentage of flights which develop component failures and can still meet the schedule for the next flight.

In order to ensure adequate fillRate is maintained across all airports, the fillRate performance indicator has to be constantly monitored so that prompt action can be taken to fine-tune the business process if the fillRate drops below an acceptable level. In our system, monitoring is carried out by the WBI Business Monitor as the CSPE emulation runs. This mimics "live" monitoring and feedback from the real world operation. Results, in the form of statistics messages, are sent to a Fault Monitor application which is available as a Web service. The WBI Business Monitor is configured to observe the Fault Monitor application. The Monitor collects data about the entities that are received by the application, compiles the data and presents customized analysis of the data. With the powerful and convenient Online Analytical Processing (OLAP) features provided by the Business Monitor, modifications can be made to the evaluation methods of the simulation results and new experimental parameters can be added with ease.

\section{EXPERIMENTS}

To study the business re-engineering process and analyze how the business process performs under different conditions, the resource management business process flow can be adjusted and changed by reordering the BPEL flow during runtime so as to maintain an acceptable fillRate. Three variations of the BPEL process flows are created to represent variations of the business process in the activity to search for replacement components.

\subsection{Alternative Business Process Flows}

There are two approaches to carry out business process re-engineering in BPEL to improve system performance. The first approach is to maintain the same business process flow, and change one or more of the Web services to other implementations. These alternative Web services may be cheaper, more efficient and robust than the original ones. The second approach involves modification to the existing business process flow by introducing or re- moving Web services from the business process as required.

In our current prototype, the first approach to business process re-engineering is adopted. Each of the three different BPEL process flows used in this study consists of the five types of Web services described in section 3.1. However, the Web service used for step iv of the process flow for searching for replacement components is different in each of the BPEL flows. The differences in the three BPEL process flows are described below:

- BPEL \#1 (flightMainProcessL.bpel): Search local inventory (L) first. If no component is available locally, it will be considered as "not-filled".

- BPEL \#2 (flightMainProcessLR.bpel): Search local inventory (L) first. If no component is available locally, proceed to book a service flight which is able to deliver a replacement from a regional inventory $(\mathrm{R})$ on time. Inventories outside the region will not be checked.

- BPEL \#3 (flightMainProcessLRW.bpel): Search local inventory (L) first. If no component is available locally, proceed to book a service flight which is able to deliver a replacement from a regional inventory $(\mathrm{R})$ or inventory outside the region (W) on time.

In our experiment, BPEL \#1 is used as the resource management application when emulating real-world operation.

\subsection{Concurrent Simulation-based Optimization}

Simulation-based decision making is a key aspect of the symbiotic simulation system. To realize this capability, the system must first be able to support concurrent simulation runs. Each simulation run would investigate the performance of a different scenario of the business process.

During normal operation with no "what-if" scenarios running, the system configuration in Figure 3 will have only a pair of CSPE flight simulator and its corresponding DS-MQ Adaptor in "Federation 0" representing an emulator of the real system. This federation is made to run in tandem with real time through a Time Manager Adapter federate (not shown in Figure 3) that regulates simulation time to scaled real time.

When the fillRate performance falls below a predefined level, the Action Manager will trigger the simulation-based optimization process. The Action Manager is a component in the WBI Monitor that reacts to situation events detected by alerting business users through emails or by invoking designated web services. In the existing prototype system, there are still some unresolved technical issues on the use of the Action Manager. Thus, triggering of the simulation-based optimization is currently carried out in the Fault Monitor application. 
Once the simulation-based optimization is triggered, different scenarios for business process re-engineering will be carried out. A pair of CSPE flight simulator and its corresponding DS-MQ Adaptor will be created for each scenario. Each pair of CSPE flight simulator and DS-MQ Adaptor will run in their individual federation, which will be independent of other federations. Each federation thus represents a different scenario that will be simulated. The use of an independent federation ensures the independent progression of simulation time and the isolation of messages sent between each federate within its own federation. This arrangement allows for concurrent execution of several simulations and is required for the symbiotic simulation system during the simulation based decision making process. Each instance of the concurrent simulations reflects the performance of a different resource management module.

Each BPEL process flow corresponding to the different scenarios is deployed as separate Web service (with a different service endpoint) in the WBI Process Server. Although there are several DS-MQ Adapters running in parallel during the optimization process, all of them still place their messages in the same three message queues. However, the message content includes an additional attribute identifying to which scenario it corresponds. The message flows in Message Broker route the message to the appropriate BPEL Web service endpoint based on the message content. The Fault Monitor Web service application and WBI Monitor will also monitor the fillRate of the different scenarios concurrently and display their relative performance.

\subsection{Experimental Results}

Although the proposed symbiotic simulation system shown in Figure 3 is still in development, we are able to carry out a set of experiments to evaluate the benefits of business process re-engineering using simulation-based optimization.

In our experiments, the configuration and inputs of the failure detection simulation model were kept the same when running with the three BPEL templates. There were 14 flights simulated with 5 destination airports with HKG, KUL and SIN belonging to region 1 and MEL and SYD belonging to region 2 . The simulation end time was set at 100,000 hours with a Mean Time Between Unscheduled Removal (MTBUR) of 500 hours. MTBUR is an accepted industry standard for reporting the reliability of a component and is defined as the total unit flying hours during a time period divided by the number of unscheduled removals of the component that occurred during the same period. Table 1 shows the fillRate for the existing operation. Tables 2 and 3 show the fillRate for the what-if scenarios.
Table 1: fillRate for BPEL \#1 - flightMainProcessL.

\begin{tabular}{|l|r|r|r|}
\hline $\begin{array}{c}\text { Destination } \\
\text { Airport }\end{array}$ & $\begin{array}{c}\text { \# of } \\
\text { Faults }\end{array}$ & $\begin{array}{c}\text { \# of } \\
\text { Filled }\end{array}$ & $\begin{array}{c}\text { fillRate } \\
\text { (\%) }\end{array}$ \\
\hline HKG & 106 & 36 & 33.96 \\
\hline KUL & 32 & 21 & 65.63 \\
\hline SIN & 259 & 131 & 50.58 \\
\hline MEL & 14 & 14 & 100.00 \\
\hline SYD & 422 & 150 & 35.55 \\
\hline
\end{tabular}

Table 2: fillRate for BPEL \#2 - flightMainProcessLR.

\begin{tabular}{|l|r|r|c|}
\hline $\begin{array}{c}\text { Destination } \\
\text { Airport }\end{array}$ & $\begin{array}{c}\text { \# of } \\
\text { Faults }\end{array}$ & $\begin{array}{c}\text { \# of } \\
\text { Filled }\end{array}$ & $\begin{array}{c}\text { fillRate } \\
\text { (\%) }\end{array}$ \\
\hline HKG & 118 & 67 & 56.78 \\
\hline KUL & 23 & 11 & 47.83 \\
\hline SIN & 238 & 146 & 61.34 \\
\hline MEL & 20 & 13 & 65.00 \\
\hline SYD & 416 & 326 & 78.37 \\
\hline
\end{tabular}

Table 3: fillRate for BPEL \#3 - fightMainProcessLRW.

\begin{tabular}{|l|r|r|r|}
\hline $\begin{array}{c}\text { Destination } \\
\text { Airport }\end{array}$ & $\begin{array}{c}\text { \# of } \\
\text { Faults }\end{array}$ & $\begin{array}{c}\text { \# of } \\
\text { Filled }\end{array}$ & $\begin{array}{c}\text { fillRate } \\
\text { (\%) }\end{array}$ \\
\hline HKG & 97 & 69 & 71.13 \\
\hline KUL & 33 & 25 & 75.76 \\
\hline SIN & 226 & 177 & 78.32 \\
\hline MEL & 12 & 9 & 75.00 \\
\hline SYD & 411 & 318 & 77.37 \\
\hline
\end{tabular}

From the tables, it can be observed that the airports with the largest number of faults within the two regions are SIN and SYD respectively. The fillRate is relatively low (SIN 50.58, SYD 35.55) in these two airports as there are not enough spare components locally (BPEL \#1 flightMainProcessL) to serve them.

Simulation-based experiments carried out using the other two BPEL process flows show that BPEL \#2 (flightMainProcessLR) and BPEL \#3 (flightMainProcessLRW) have an improved fillRate for airports SIN and SYD. This is because of the searching process defined by the BPEL flow. In the BPEL \#2, if a local spare component cannot be found, the search will continue to look for a service flight which can bring a spare from any other airports within the region. This can be observed from the pulling effect on KUL and MEL which causes their fillRates to drop somewhat. However, the net change in fillRate within the region is positive and is more balanced. For BPEL \#3, the search process will attempt to find a service flight which can bring a spare component from any airport worldwide. By using any one of these two BPEL processes, the chance that a service flight is able to be found and the replacement is able to be delivered on time would be higher, compared to the existing operation using BPEL \#1. 


\section{CONCLUSION AND FUTURE WORK}

In this paper, we describe our proposed framework for a symbiotic simulation system for carrying out business process re-engineering using the IBM WebSphere Suite. Our study shows that the service oriented approach to deploying business application can allow for flexible deployment and reconfiguration to adapt to changes in the operating environment. We will expand the existing case study to include the evaluation of Web services that implement different re-balancing strategies for spare components across all airports.

We are currently in the process of developing a generic symbiotic framework that can be applied to different domains such as manufacturing, maritime, aerospace and defence. Our generic symbiotic simulation framework will allow for not just reactive simulation, but also proactive as well as reflective simulation to be carried out to better anticipate possible events in the system as well as learn from past events.

\section{ACKNOWLEDGMENTS}

This work is carried out under the IBM Shared University Research Grant awarded to NTU and SIMTech.

\section{REFERENCES}

Carnahan, J.C., P. F. Reynolds, and D. C. Brogan. 2004. Visualizing Coercible Simulations, In Proceedings of the 2004 Winter Simulation Conference. 411-419. Washington, USA.

DMSO. 2002. RTI 1.3-Next Generation Programmer's Guide Version 5. DoD, DMSO.

Fujimoto, R., D. Lunceford, E. Page, and A. Uhrmacher. 2002. Technical Report of the Dagstuhl-Seminar Grand Challenges for Modelling and Simulation. Available

via

<www.dagstuhl.de/02351/Report> [accessed June 13, 2007].

IBM, 2007. WebSphere Business Process Management software: understand, define, execute and optimize. Available via

<www.ibm.com/software/integration> [access June 13 2007].

IEEE 1516, 2000. IEEE Standard for Modeling and Simulation (M\&S) High Level Architecture (HLA). New York, NY: Institute of Electrical and Electronics Engineers.

Kuhl, F., R. Weatherly, and J. Dahmann. 1999. Creating Computer Simulation Systems: An Introduction to the High Level Architecture. Prentice Hall PTR.

Lendermann, P., S. J. Turner, M. Y. H. Low, B. P. Gan, N. Julka, L. P. Chan, W. T. Cai, L. H. Lee, E. P. Chew, S. Y. Teng, and L. F. McGinnis. 2007. An In- tegrated and Adaptive Decision-Support Framework for High-Tech Manufacturing and Service Networks. Journal of Simulation. 1(2): 69-79.

Leymann, F. and D. Roller. 2002. "Processes in a Web Services world". Available via: <www128.ibm.com/developerworks/webservi ces/library/ws-bpelwp> [accessed June 13 2007].

Liu, X., P. Reynolds, and D. Brogan. 2006 Using Abstraction in the Verification of Simulation Coercion. In Proceedings of the 20th Workshop on Principles of Advanced and Distributed Simulation (PADS). 119128. Singapore.

Loitiere, Y., D. C. Brogan, and P. F. Reynolds. 2004. Simulation Coercion Applied to Multiagent DDDAS. In Proceedings of the 2004 International Conference on Computational Science (ICCS), 789-796. Krakow, Poland.

Low, M. Y. H., L. K. Wei, P. Lendermann, S. J. Turner, S. Leo, and R. Chim. 2005. An Agent-based Approach for Managing Symbiotic Simulation of Semiconductor Assembly and Test Operations. In Proceedings of the 2005 International Conference on Autonomous Agent and Multiagent Systems (AAMAS), 85-92. Utrecht, The Netherland.

Low, M. Y. H., and S. J. Turner. 2006. Using Web Services and Business Process Execution Language for HLA-based Distributed Supply-chain Simulation. In Proceedings of the UK Operational Research Society Simulation Workshop 2006 (SW06), 23-31. UK.

Wang, X. G., S. J. Turner, S. J. E. Taylor, M. Y. H. Low, and B. P. Gan. 2005. A COTS Simulation Package Emulator (CSPE) for Investigating COTS Simulation Package Interoperability. In Proceedings of the 2005 Winter Simulation Conference, 402-411, Orlando, FL USA.

\section{AUTHOR BIOGRAPHIES}

MALCOLM YOKE HEAN LOW is an Assistant Professor in the School of Computer Engineering at the Nanyang Technological University (NTU), Singapore. Prior to this he was with the Singapore Institute of Manufacturing Technology (SIMTech). He received his Bachelor and Master of Applied Science in Computer Engineering from NTU in 1997 and 1999 respectively. He was awarded a Gintic (now SIMTech) Postgraduate Scholarship in 1999. In 2002, he received his D.Phil. degree in Computer Science from Oxford University. His current research interest is in the application of parallel/distributed simulation, grid computing and agent technology for the modeling, simulation, analysis and optimization of complex systems. His e-mail is <yhlowentu.edu.sg $>$. 
STEPHEN J. TURNER joined Nanyang Technological University (NTU), Singapore, in 1999 and is Director of the Parallel and Distributed Computing Centre in the School of Computer Engineering. Previously, he was a Senior Lecturer in Computer Science at Exeter University (UK). He received his MA in Mathematics and Computer Science from Cambridge University (UK) and his MSc and $\mathrm{PhD}$ in Computer Science from Manchester University (UK). His current research interests include parallel and distributed simulation, distributed virtual environments, grid computing and multi-agent systems. He is the steering committee chair of the Workshop on Principles of Advanced and Distributed Simulation and an advisory committee member of the Distributed Simulation and Real Time Applications Symposium. His email address is <assjturnerentu.edu.sg>.

DING LING is a senior undergraduate student at the Nanyang Technological University (NTU), Singapore.

HAI LIANG PENG is a senior undergraduate student at the Nanyang Technological University (NTU), Singapore.

LAI PENG CHAN is a Senior Research Engineer with the Planning and Operations Management Group at the Singapore Institute of Manufacturing Technology. She obtained her BSc and MTech at the National University of Singapore. Her research interests include modeling and simulation, optimization and intelligent systems. Her email address is <lpchandsimtech.astar.edu.sg>.

PETER LENDERMANN is the Co-Founder and CEO of D-SIMLAB Technologies, a Singapore-based company providing simulation-based decision support solutions and services to Aerospace, Semiconductor Manufacturing and other asset-intensive industries. He has been engaged in the simulation community since the early 1990's when he worked in a multinational research collaboration at the European Laboratory for Particle Physics CERN (Geneva, Switzerland) and Nagoya University (Japan). In 1996 he joined a German consulting firm where he was responsible for business process re-engineering projects with numerous process manufacturing, aviation and automotive clients in Europe, Canada and China. In 2000 he joined the Singapore Institute of Manufacturing Technology where he led the simulation-related research activities until spinning them off into D-SIMLAB Technologies. Peter holds a PhD in Applied High-Energy Physics from Humboldt-University in Berlin (Germany) and an MBA in International Economics and Management from SDA Bocconi in Milan (Italy). He is also an Adjunct Associate Professor at the Department of Industrial and Systems Engineering at the National University of Singapore. His email address is petered-simlab.com>.
STEVE BUCKLEY has been a Research Staff Member at the IBM Thomas J. Watson Research Center in Yorktown Heights, NY since 1987, and a manager at that facility since 1995 . His most recent interest is in Sense \& Respond systems; his team has implemented Sense \& Respond for several IBM lines of business. He was one of a group of IBM researchers who received the prestigious Franz Edelman award from INFORMS in 1999 for the successful deployment of supply chain simulation and optimization technology in IBM. He received the Ph.D. degree in Computer Science from MIT in 1987. His e-mail address is <sbuckleyeus.ibm. com>. 